\title{
Alterations in glutathione, nitric oxide and 3-nitrotyrosine levels following exercise and/or hyperbaric oxygen treatment in mice with diet-induced diabetes
}

\author{
LUIS ROGELIO GUTIÉRREZ-CAMACHO, ALEXANDRE KORMANOVSKI, \\ MARÍA DEL CARMEN CASTILLO-HERNÁNDEZ, GUSTAVO GUEVARA-BALCÁZAR \\ and ELEAZAR LARA-PADILLA
}

Instituto Politécnico Nacional, Escuela Superior de Medicina, Mexico City 11340, Mexico

Received June 4, 2019; Accepted November 29, 2019

DOI: 10.3892/br.2020.1291

\begin{abstract}
Oxidative stress is involved in the development of diabetes. Nitric oxide (NO) contributes to oxidative stress, affects the synthesis of glutathione (GSH) in tissues and also regulates important physiological processes. The levels of nitrosative stress, assessed by measuring the levels of 3-nitrotirosina (3NT) as well as the bioavailability of $\mathrm{NO}$ are modulated by exercise and hyperbaric oxygenation (HBO). The aim of the present study was to evaluate the effects of exercise and $\mathrm{HBO}$ on the levels of NO, 3NT and GSH in tissues of various organs obtained from diabetic mice. Female mice were fed a high-fat/high-fructose diet to induce diabetes. Mice with diabetes were subjected to exercise and/or HBO. Initial and final concentrations of $\mathrm{NO}$, $3 \mathrm{NT}$ and GSH were assessed in the muscle, liver, kidney, heart, spleen, lung, brain, visceral adipose, thoracic aorta and small intestine. Diabetes did not affect initial values of NO, although it significantly increased the levels of 3NT. The basal level of GSH in the diabetic group was lower than or comparable to that of the control group in the majority of the organs assessed. A negative correlation was observed between $3 \mathrm{NT}$ and GSH levels in the initial values of all tissues of the control group only, whereas all pathological tissues showed a positive correlation between $\mathrm{NO}$ and GSH. There was an increase or a stabilization of GSH levels in the majority of the organs in all treated mice despite the increase in nitrosative stress.
\end{abstract}

\section{Introduction}

Nitric oxide (NO) acts as an intracellular messenger in physiological and pathological conditions (1). The bioavailability

Correspondence to: Professor Alexandre Kormanovski, Instituto Politécnico Nacional, Escuela Superior de Medicina, Mexico City 11340, Mexico

E-mail: kormanovski@yahoo.com.mx

Key words: diabetes, nitric oxide, glutathione, exercise, hyperbaric oxygenation, mice of NO, and therefore its ability to perform physiological functions, can be affected by the excessive production of reactive oxygen species (ROS) involved in the development of diabetes. ROS have the capacity to reduce the enzymatic (2) and non-enzymatic (3) production of $\mathrm{NO}$, or simply eliminate it from tissues. NO reacts with superoxide radicals resulting in peroxynitrite, a very potent oxidant that reacts with tyrosine residues of proteins to form 3-nitrotirosine (3NT). 3NT serves as an indicator of nitrosative stress, the elimination of NO or modulation of signaling pathways. According to studies using animal models, NO decreases the harmful effects of ROS on biomolecules, whereas ROS limits the effects of NO by eliminating it (4) or affecting its intracellular receptors (5). Diabetes and its complications increase dysfunction of the endothelium (6), the possible mechanisms of which are discussed in a recent review $(7)$.

In general, the bioavailability of NO is diminished during the pathogenesis of diabetes (7-9), despite an increase in the plasma levels in patients, as was shown by a meta-analysis (10). This limited bioavailability may be due to the elimination of NO by the superoxide radical (11), or by modulation of its production through enzymatic mechanisms (12) and/or nonenzymatic conditions such as ischemia or stomach acidity $(13,14)$.

Glutathione (GSH) is an abundant endogenous antioxidant synthesized in large quantities in the liver. It is transported via blood flow to tissues when there is an imbalance between the production of ROS and the endogenous antioxidant mechanisms. Extracellular GSH is not able to pass through cell membranes; however, following enzymatic breakdown into its constituent amino acids, these amino acids are able to traverse the membrane. Once inside the cell, the amino acids are available for the resynthesis of GSH. GSH is irreversibly utilized when its product of oxidation (GSSG) is not recycled into GSH by glutathione reductase (15). The erythrocyte GSH levels are lower in patients with type 2 diabetes $(16,17)$ and there are reports that have shown that GSH production gradually decreases in patients with diabetes with complications $(18,19)$, and that this decrease is dependent on the degree of hyperglycemia (20). In a recent study it was shown that a high-fat diet resulted in GSH deficiency in the kidney of mice (21). GSH has a high affinity for NO, and S-nitrosoglutathione is the primary 
intermediary metabolite in the production of other nitrosothiols which serve as metabolic regulators $(22,23)$. Based on an in vivo study using endotoxemic mice, an increase in NO resulted in decreased GSH synthesis, whereas a decrease in NO levels had the opposite effect (24).

Exercise prevents the harmful effects of ROS, and NO may be a central signaling molecule involved in the adaptation of muscles to exercise (25). A recent review analyzed the evidence showing the protective effects of aerobic exercise against the development of complications in patients with diabetes (26). Hyperbaric oxygenation (HBO) shows a beneficial therapeutic effect in patients with ulcers caused by diabetes; however, the underlying mechanisms have not been fully resolved. Pre-treatment with $\mathrm{HBO}$ exhibits a protective effect against oxidative stress in different types of tissues in animal models of various pathological processes or stress (27-31). Several studies have explored the hypothesis that oxidative stress serves an important role in $\mathrm{HBO}$-mediated effects at the cellular level $(32,33)$. However, studies examining the effects of HBO on NO metabolism are limited: An increase in pulmonary (34) or cardiac levels of NO (35) results in a vasodilatory effect, and thus an increase of oxygen being delivered to tissues (36). The aim of the present study was to evaluate the levels of NO, 3NT and GSH in mice with type 2 diabetes induced by a high-fat/high-fructose (HFHF) diet, compared with control animals, and the effects of exercise and/or HBO on NO, 3NT and GSH levels in the diabetic mice.

\section{Materials and methods}

Animals and study design. A total of 70 3-month-old female CD1 mice (average weight, $35.8 \mathrm{~g}$ ) were housed in acrylic cages $(20 \times 20 \times 30 \mathrm{~cm})$ with a 12:12 h light/dark cycle. Mice pups were used as adult female rodents and humans have a similar pattern of growth, in which weight is stabilized at the adult stage (37). The synchronization of the estrous cycle in the mice was not taken into consideration. In the first 3 months (phase 1), animals were fed a normal (control group) or a HFHF diet in (diabetes group) and parameters were measured in samples taken at the end of the 3 months and were used as the initial values. The use of drinking liquids that contained fructose results in a high fasting levels of glucose in the animals (38). Therefore, these mice were included in the high-fat diet. At the end of the second 3-month period (phase 2), samples were taken again from the untreated groups (final values) and from the experimental groups: After exercise training, $\mathrm{HBO}$ or a combination of these two treatments. The treatments were compared to the untreated diabetic mice. The final values of the control mice were used to examine the effects of ageing between the two phases. The time frame of the protocol and the animal groups are illustrated in Table I.

The percentage composition of the normal diet (Chow), consumed by the control mice was $9 \%$ fat, $29 \%$ protein and $62 \%$ carbohydrates. For the high-fat diet, the normal solid food was enriched with lard baked at $100^{\circ} \mathrm{C}$, resulting in percentage composition of micronutrients of $38 \%$ fat, $19 \%$ protein and $43 \%$ carbohydrates. The high-fructose diet consisted of $40 \%$ carbohydrates (30\% fructose and $10 \%$ dextrose) in a solution of water. Food and water were provided ad libitum for the control group and water with fructose and a HF diet for the
Table I. Time frame of the protocol used and the different groups used.

\begin{tabular}{lcc}
\hline Animal groups & Phase $1^{\mathrm{a}}$, diet & Phase $2^{\mathrm{b}}$, treatment \\
\hline Control $^{\mathrm{c}}$ & Normal & - \\
Diabetes $^{\mathrm{c}}$ & HFHF & - \\
Control & Normal & Untreated \\
Diabetes & HFHF & Untreated \\
Diabetes & HFHF & Exercise \\
Diabetes & HFHF & HBO \\
Diabetes & HFHF & Exercise + HBO
\end{tabular}

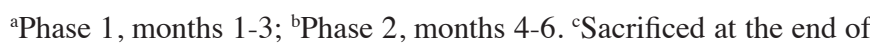
phase 1 . HFHF, high fructose, high fat; HBO, hyperbaric oxygenation.

experimental group. Weight and body composition (Lee index: $\sqrt[3]{g / l}$; where $g$ is the weight in grams and 1 is the length of mice in $\mathrm{mm}$ ) were evaluated at the beginning and end of phase 2. Glucose tolerance was quantified at the end of phase 1 by comparing the diabetic group to the control group. A solution of $60 \%$ dextrose was injected intraperitoneally at a dose of $3 \mathrm{~g} / \mathrm{kg}$. The glucose concentration in the blood was measured after $0,15,30,60,90$ and 120 min by taking blood samples from the tail.

At the start of phase 2, 7 mice from each group (control and diabetes) were sacrificed by the intraperitoneal injection of pentobarbital $(100 \mathrm{mg} / \mathrm{kg})$ to determine the initial values of parameters in different tissues. During phase 2, the animals in the control and diabetes groups were fed with their respective diets. The total duration of the experiment was 6 months.

Treatments. The exercise protocol started with a 2 -week adaptation period of swimming (flotation) sessions 3 times per week. The time of each session was gradually increased from 10 to $60 \mathrm{~min}$. For the following 12 weeks, the mice were trained 3 times per week, $60 \mathrm{~min}$ per session. The present protocol was designated as moderate exercise training, based on a comparison to other rodent studies using swimming training $(39,40)$. The water temperature was $32 \pm 2^{\circ} \mathrm{C}$. For the swimming sessions, animals were placed in transparent tanks divided into cells $(25 \times 25 \mathrm{~cm})$, one animal per cell. Following each exercise session, mice were dried and returned to their cages. All sessions were conducted between 11 am and $1 \mathrm{pm}$. The HBO procedure was carried out from 9 am to 10:30 am in a hyperbaric chamber for small animals, using an oxygen pressure of 2 ATA (15 min pressurization, $60 \mathrm{~min}$ exposure and 15 min depressurization). A total of $10 \mathrm{HBO}$ sessions were performed, once every other day, in the second month of phase 2, as the therapeutic use of HBO in humans includes 10-20 sessions (32). Upon completion of $\mathrm{HBO}$ and/or exercise, animals were returned to their cages and fed with their corresponding diet.

Tissue processing. The values of the measured parameters were assessed in tissues extracted from the liver, skeletal muscle (the vastus lateralis from the hind leg), lung, heart, thoracic aorta, brain, spleen, small intestine (lamina propria and mucosa), 

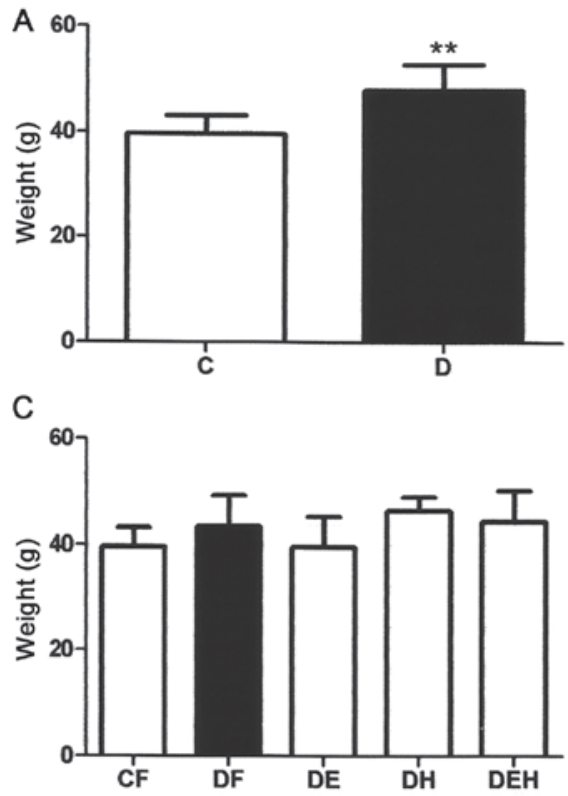

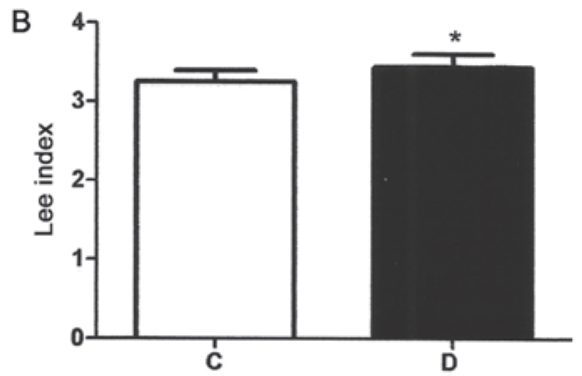

D

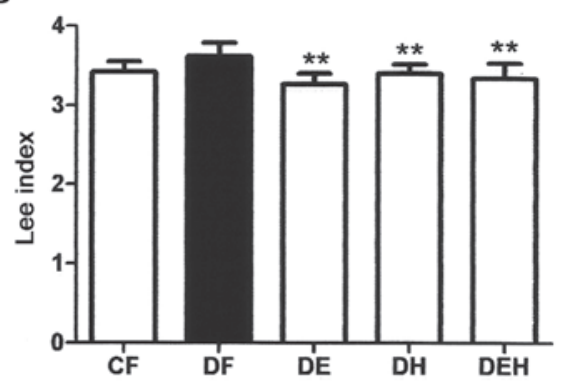

Figure 1. Weight and body composition of the animals in the control and experimental groups. (A) Initial body weight and (B) body composition. (C) Final body weight and (D) body composition. Data are presented as the mean \pm standard deviation. ${ }^{*} \mathrm{P}<0.05,{ }^{* *} \mathrm{P}<0.01 \mathrm{vs}$. $\mathrm{C}$ or $\mathrm{DF}$. C, initial values in the control group; D, initial values in the diabetic group; $\mathrm{CF}$, final values in the control group; DF, final values in the diabetic group; DE, values in the diabetic mice treated with exercise; $\mathrm{DH}$, values in diabetic mice treated with hyperbaric oxygenation; DEH diabetic mice treated with both exercise and hyperbaric oxygenation.

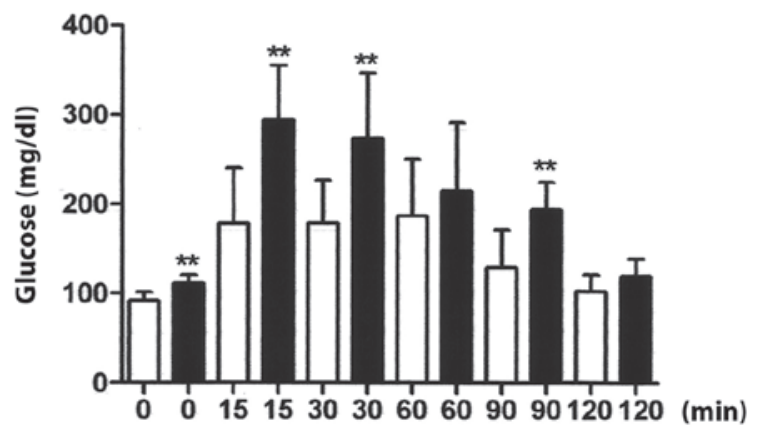

Figure 2. Glucose tolerance curves of mice in the $\mathrm{C}$ and $\mathrm{D}$ groups at the end of phase $1 .{ }^{* *} \mathrm{P}<0.01$. C, control; $\mathrm{D}$, diabetic.

kidney and visceral adipose tissue. The aforementioned parameters were assessed in these tissues for the following physiological reasons: To evaluate the possible redistribution of GSH from the liver to other tissues during stress, and to examine whether there was a relation between redistribution and the response to NO. Samples were obtained by placing a tissue in $30 \mathrm{mmol}$ cold PBS (pH 7.2) and adding $0.1 \%$ of Triton X-100 ( $1 \mathrm{mg}$ of tissue per $10 \mu \mathrm{l}$ buffer). Tissues were homogenized and centrifuged at $900 \mathrm{x} \mathrm{g}$. for $15 \mathrm{~min}$ at $4^{\circ} \mathrm{C}$ and the supernatants were stored at $-80^{\circ} \mathrm{C}$ for processing within two weeks. Cayman Chemical Company chemical kits were used to measure total proteins (TP; cat. no. 704002), NO (nitrate/nitrite colorimetric assay kit; cat. no. 780001) and total reduced GSH (GSH assay kit; cat. no. 703002) in the tissue homogenates. In the tissues with a significant presence of blood (liver, heart and kidney), the homogenates were treated with an Amicon ultra- 0.5 centrifugal filter device $(30 \mathrm{~K})$ prior to measurement of NO levels. The levels of 3NT (3NT ELISA kit; Abcam; cat. no. ab116691) was measured in the homogenates using ELISA. The values of NO, GSH and 3NT are expressed as nmol/mg of TP.
Statistical analysis. GraphPad Prism version 5 (GraphPad Software, Inc.) was used for statistical analysis. The mean values and standard deviations were obtained for each group. The count data were analyzed by ANOVA (7 animals/group), followed by a post-hoc Tukey's test. Bivariate Pearson's correlation analysis was used to assess the relation between the parameters in all tissues. $\mathrm{P}<0.05$ was considered to indicate a statistically significant difference.

\section{Results}

Lee index. For all animals, the Lee index and weight was determined at the start (Fig. 1A and B) and end of phase 2 (Fig. 1C and D). During phase 1, a significant gain in weight was observed for the animals fed the HFHF diet $(\mathrm{P}<0.01$; Fig. 1A). The inflammatory response promotes insulin resistance, which is manifested as weight gain and an accumulation of body fat (41). The Lee index, which has a positive correlation with body fat in females with obesity (42), and is an indicator of type 2 diabetes, was significantly higher in the diabetic mice compared with the control mice (Fig. 1B). At the end of phase 2, a significant decrease in the Lee index was observed for all treatments $(\mathrm{P}<0.01$; Fig. 1D).

To analyze the behavior of the tolerance curve for glucose (Fig. 2), it is important to take into account, the measurement at 15 min captures the rapid release of insulin into the system. This measurement provides the first evidence of pancreatic failure. It is also a crucial element in the diagnosis of type 2 diabetes $(38,43,44)$. The results confirm the successful establishment of diet-induced diabetes mellitus type 2 (Figs. 1 and 2). NO, 3NT and GSH levels were evaluated in tissues from various organs at the beginning and end of phase 2 to determine the effect of the treatments with exercise and/or HBO on the diabetic mice. The results of the measured parameters in all tissues are presented in Table II. 

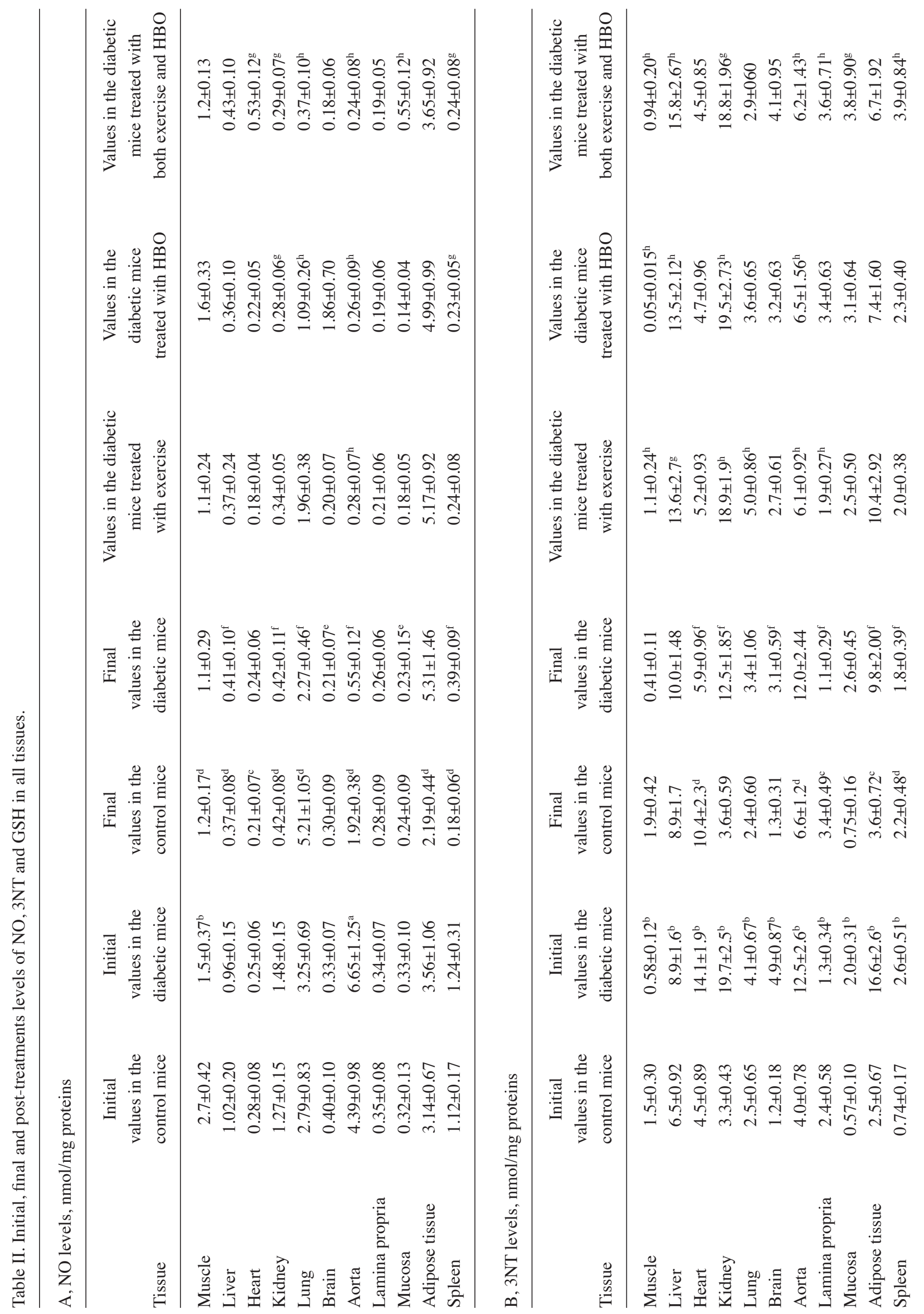
Muscle. The basal level of NO in the muscle tissues of mice with diabetes was $\sim 50 \%$ of the levels observed in the control mice; whereas the final level of $\mathrm{NO}$ in the muscle tissue of the control group was $<50 \%$ of the basal value, and a similar decrease was observed in the diabetic mice between the start and end of phase 2. The values for NO in the control and diabetic mice at the end of phase 2 did not differ significantly. Compared with the untreated diabetes group, no significant differences were observed in the levels of NO in the animals treated with exercise, HBO or both. The development of diabetes caused a 2.5 -fold decrease in 3NT levels compared with the control group, similar to the decrease in NO levels in muscle tissue between the two groups. In both groups, the final levels of 3NT remained relatively stable at the end of study, but were lower in the diabetic mice. Additionally, 3NT levels increased to a similar degree in the mice subjected to exercise or exercise and HBO compared with the untreated diabetic mice. For the animals treated with $\mathrm{HBO}$ alone, there was a significant decrease in $3 \mathrm{NT}$ levels to a minimal level, corroborating the protective effect of $\mathrm{HBO}$ against nitrosative stress in muscle tissue $(4,6)$. The effect of exercise was more prominent in the mice treated with exercise and HBO, as the response to exercise training alone was similar to the combined treatment. The basal levels of GSH in muscle tissues of mice fed either diet were high and did not differ significantly. The final levels of GSH were $>10$-fold lower in both groups, with the lowest level observed in the mice with diabetes. All treatments resulted in a significant increase in GSH levels compared with the final values in the diabetic mice. The effect of treatment with HBO was more prominent than with exercise, and the results coincided with the decrease in 3NT levels in the muscle tissues.

Liver. The initial levels of NO in liver tissue was not affected by diabetes; whereas the final levels of NO in the liver decreased $>50 \%$ in both groups. In the animals with diabetes, NO levels were not altered by any of the treatments. The mice fed a HFHF diet had higher initial 3NT levels compared with the mice fed a normal diet, confirming an increased basal level of nitrosative stress in a diabetic state. The final levels of 3NT in the control group increased moderately $(\mathrm{P}<0.05)$, but no significant difference was observed in the final values between the groups. In comparison to the final values of the diabetic group, the three treatments increased the levels of 3NT to a similar degree (all $\mathrm{P}<0.05$ ). Therefore, each treatment increased nitrosative stress in the liver. The initial levels of GSH in the liver was $>30$-fold higher compared with muscle tissues, but was not significantly different between the diabetic mice compared with the control group. No significant differences were observed in the final levels of GSH between the two groups, and none of the three treatments resulted in significant differences in levels.

Heart. The initial levels of NO were similar in the heart tissues of both groups. The final values of NO were moderately $(\mathrm{P}<0.05)$ reduced in the control animals and there was no significant change in the diabetic mice. Exercise reduced the NO levels, but $\mathrm{HBO}$ alone had no effect. There was large and significant increase in the levels of $\mathrm{NO}$ in the mice treated with both exercise and HBO, showing a possible synergistic effect of exercise and HBO on the heart which was absent in either treatment alone. The initial levels of 3NT were low 
in the control mice and $>3$-fold higher for the diabetic mice. Compared with the initial values, the final levels of 3NT were increased in the control mice and decreased in the diabetic mice (both $\mathrm{P}<0.01$ ). Neither exercise, HBO or both had an effect on $3 \mathrm{NT}$ levels in the heart of diabetic mice, suggesting that these treatments did not affect nitrosative stress. The development of diabetes did not significantly affect the initial levels of GSH in the heart, although the GSH levels were elevated in both groups in the majority of other tissues. The elevated initial levels of GSH observed in all mice was followed by a significant and large decrease in the final levels in both groups (all $\mathrm{P}<0.01$ ). The final levels were lower in the diabetic mice compared with the control mice $(\mathrm{P}<0.01)$. All treatments increased GSH levels in diabetic mice to levels similar to those observed in the final values of the control group.

Kidney. The initial levels of NO in kidney tissues was comparable between the control and diabetic mice. A $~ 3$-fold decrease in NO levels was observed in the final values in both groups and all treatments moderately reduced NO levels, and treatment with $\mathrm{HBO}$ or exercise and $\mathrm{HBO}$ combined resulted in a significant reduction in NO levels. In the control group, the initial and final values of $3 \mathrm{NT}$ levels were very low. However, the initial value in the diabetes group was $\sim 17$-fold higher compared with the control. Despite the decrease in $3 \mathrm{NT}$ levels in the final values in both groups, the levels in the diabetic mice were still 4-fold higher than the control group. The three treatments increased the levels of $3 \mathrm{NT}$ to a similar degree, reaching levels similar to those of the initial values of the diabetic mice. The initial GSH levels were moderately but not significantly lower in the diabetic group $(\mathrm{P}>0.05)$. The final levels of GSH were significantly and drastically reduced compared with the minimal levels in both the untreated groups $(\mathrm{P}<0.01)$. All treatments increased this parameter compared with the final values in the diabetic group, with the combined treatment having the most prominent effect, followed by HBO alone and then exercise alone. With both treatments involving $\mathrm{HBO}$, the final levels of GSH were comparable to the initial values of the mice with diabetes. Thus, nitrosative stress was exacerbated in the kidney due to the development of diabetes and with the three treatments, similar to what was observed in the liver. The initial levels of GSH in the kidney in both groups was the lowest compared with all tissues assessed in the present study, consistent with a previous study (45).

Lung. In lung tissues, diabetes did not affect the initial levels of $\mathrm{NO}$, and the final levels of $\mathrm{NO}$ were increased in the control group $(\mathrm{P}<0.01)$ and decreased in the diabetes group $(\mathrm{P}<0.01)$, compared with the initial levels. Both treatments involving HBO led to a decrease, and the decrease was more pronounced in the combined treatment group. Lung tissue is the first to be affected by sessions of HBO. The initial levels of 3NT were higher in the diabetic mice, and no significant change was observed in the final values in either the control or diabetic mice. Exercise increased the levels of 3NT, whereas HBO and the combined treatment did not have a significant effect. The changes in GSH levels in lung tissues were similar to those observed in other tissues. The initial values of the control and diabetic mice were comparable, and the final values were significantly decreased in both groups compared to the respective initial value, but did not differ significantly between groups. HBO reduced GSH levels, whereas exercise and the combined treatment did not have a notable effect.

Thoracic aorta. The initial levels of NO in the thoracic aorta were higher in the diabetic mice compared with the control mice $(\mathrm{P}<0.05)$. The final levels were significantly and drastically lower in both groups $(\mathrm{P}<0.01)$, and the decrease in the diabetic mice was greater. All treatments resulted in $~ 50 \%$ decrease in the NO levels. The initial levels of $3 \mathrm{NT}$ were higher in the diabetic group compared with the control group $(\mathrm{P}<0.01)$. The final levels of $3 \mathrm{NT}$ levels were increased in the control group only compared with the respective initial values (all $\mathrm{P}<0.01$ ). All treatments decreased the increased levels of $3 \mathrm{NT}$ in the diabetic mice to a similar degree (all $\mathrm{P}<0.01$ ). The levels of GSH were higher in the diabetic mice compared with the control group $(\mathrm{P}<0.05)$. In both groups, the final GSH levels were considerably lower compared with the respective initial values (both $\mathrm{P}<0.01$ ). Exercise or $\mathrm{HBO}$ significantly decreased GSH levels (both $\mathrm{P}<0.01$ ), and to a lesser extent in the mice treated with the combined treatment.

Intestinal lamina propria. The development of diabetes did not significantly affect the initial, final or post-treatments values in the lamina propria. The initial levels of 3NT in the lamina propria were lower in the diabetic mice compared with the control group $(\mathrm{P}<0.01)$. For the control mice, there was an increase in the levels of $3 \mathrm{NT}$ between the initial and final levels $(\mathrm{P}<0.05)$, whereas in the diabetic group there was no significant change. The decreased levels of $3 \mathrm{NT}$ in the diabetic mice were increased with exercise $(\mathrm{P}<0.01)$, and the increase was greater in the mice treated with $\mathrm{HBO}$ alone or the combined treatment (both $\mathrm{P}<0.01$ ), reaching similar values to the final levels of the control group. This confirmed the predominance of the effects of HBO over exercise in the exacerbation of nitrosative stress. The initial levels of GSH were higher in the mice diabetic mice compared with the control animals $(\mathrm{P}<0.01)$. The final levels of GSH decreased in the control group and in the diabetic group $(\mathrm{P}<0.05)$, but remained higher in the diabetic mice compared with the control mice $(\mathrm{P}<0.05)$. The two treatments involving exercise significantly reduced the levels of GSH (both $\mathrm{P}<0.01$ ); whereas HBO alone did not notably affect GSH levels, demonstrating the predominance of exercise in regard to this parameter.

Mucosa. The initial levels of NO were not affected by diabetes. Only final levels of NO in the diabetic group decreased in intestinal mucosa $(\mathrm{P}<0.05)$, and only combined treatment increased this parameter $(\mathrm{P}<0.01)$. The initial levels of $3 \mathrm{NT}$ were relatively low in the mucosa of control mice and was higher in the diabetic group $(\mathrm{P}<0.01)$. There were no significant changes in the final values of $3 \mathrm{NT}$ in both groups. $3 \mathrm{NT}$ levels were not significantly affected by exercise or HBO treatment alone, the combination treatment resulted in an increase in $3 \mathrm{NT}$ levels $(\mathrm{P}<0.05)$, suggesting a synergistic effect of these two treatments. The similar initials levels of GSH were followed by a sharp decrease in the final values of both groups in the mucosa. Exercise increased the levels of GSH in the mucosa $(\mathrm{P}<0.01)$, whereas the combined treatment decreased this parameter. 
Table III. Direction of significant changes of measured parameters in all tissues based on Table II.

A, Animal model

\begin{tabular}{|c|c|c|c|c|c|c|c|c|c|}
\hline \multirow[b]{2}{*}{ Tissue } & \multicolumn{3}{|c|}{$\begin{array}{c}\text { Initial levels, } \\
\text { Control vs. diabetic group }\end{array}$} & \multicolumn{3}{|c|}{$\begin{array}{l}\text { Control, } \\
\text { final vs. initial levels }\end{array}$} & \multicolumn{3}{|c|}{$\begin{array}{l}\text { Diabetic group, } \\
\text { final vs. initial levels }\end{array}$} \\
\hline & $\mathrm{NO}$ & $3 \mathrm{NT}$ & GSH & $\mathrm{NO}$ & $3 \mathrm{NT}$ & GSH & $\mathrm{NO}$ & $3 \mathrm{NT}$ & GSH \\
\hline Muscle & D & $\mathrm{D}$ & 0 & $\mathrm{D}$ & 0 & $\mathrm{D}$ & 0 & 0 & $\mathrm{D}$ \\
\hline Liver & 0 & I & 0 & $\mathrm{D}$ & 0 & 0 & $\mathrm{D}$ & 0 & 0 \\
\hline Heart & 0 & I & 0 & $\mathrm{D}$ & I & $\mathrm{D}$ & 0 & $\mathrm{D}$ & $\mathrm{D}$ \\
\hline Kidney & 0 & I & 0 & $\mathrm{D}$ & 0 & $\mathrm{D}$ & $\mathrm{D}$ & $\mathrm{D}$ & D \\
\hline Lung & 0 & I & 0 & I & 0 & $\mathrm{D}$ & $\mathrm{D}$ & 0 & $\mathrm{D}$ \\
\hline Brain & 0 & I & 0 & 0 & 0 & $\mathrm{D}$ & $\mathrm{D}$ & $\mathrm{D}$ & $\mathrm{D}$ \\
\hline Aorta & I & I & I & $\mathrm{D}$ & I & $\mathrm{D}$ & $\mathrm{D}$ & 0 & $\mathrm{D}$ \\
\hline LP & 0 & $\mathrm{D}$ & I & 0 & I & $\mathrm{D}$ & 0 & 0 & D \\
\hline Muscosa & 0 & I & 0 & 0 & 0 & $\mathrm{D}$ & $\mathrm{D}$ & 0 & $\mathrm{D}$ \\
\hline AT & 0 & I & $\mathrm{D}$ & $\mathrm{D}$ & I & 0 & 0 & $\mathrm{D}$ & 0 \\
\hline Spleen & 0 & I & $\mathrm{D}$ & $\mathrm{D}$ & I & $\mathrm{D}$ & $\mathrm{D}$ & $\mathrm{D}$ & $\mathrm{D}$ \\
\hline
\end{tabular}

B, Treatment

\begin{tabular}{|c|c|c|c|c|c|c|c|c|c|}
\hline \multirow[b]{2}{*}{ Tissue } & \multicolumn{3}{|c|}{$\mathrm{NO}$} & \multicolumn{3}{|c|}{$3 \mathrm{NT}$} & \multicolumn{3}{|c|}{ GSH } \\
\hline & $\mathrm{DE}$ & $\mathrm{DH}$ & $\mathrm{DEH}$ & $\mathrm{DE}$ & $\mathrm{DH}$ & $\mathrm{DEH}$ & $\mathrm{DE}$ & $\mathrm{DH}$ & $\mathrm{DEH}$ \\
\hline Muscle & 0 & 0 & 0 & I & D & I & I & I & I \\
\hline Liver & 0 & 0 & 0 & I & I & I & 0 & 0 & 0 \\
\hline Heart & 0 & 0 & I & 0 & 0 & 0 & I & I & I \\
\hline Kidney & 0 & $\mathrm{D}$ & $\mathrm{D}$ & I & I & I & I & I & I \\
\hline Lung & 0 & $\mathrm{D}$ & $\mathrm{D}$ & I & 0 & 0 & 0 & $\mathrm{D}$ & 0 \\
\hline Brain & 0 & 0 & 0 & 0 & 0 & 0 & 0 & I & $\mathrm{D}$ \\
\hline Aorta & $\mathrm{D}$ & $\mathrm{D}$ & D & $\mathrm{D}$ & $\mathrm{D}$ & $\mathrm{D}$ & $\mathrm{D}$ & $\mathrm{D}$ & 0 \\
\hline LP & 0 & 0 & 0 & $\mathrm{I}$ & I & I & $\mathrm{D}$ & 0 & $\mathrm{D}$ \\
\hline Muscosa & 0 & 0 & I & 0 & 0 & I & I & 0 & $\mathrm{D}$ \\
\hline AT & 0 & 0 & 0 & 0 & 0 & 0 & 0 & 0 & $\mathrm{D}$ \\
\hline Spleen & $\mathrm{D}$ & $\mathrm{D}$ & D & 0 & 0 & I & I & $\mathrm{D}$ & I \\
\hline
\end{tabular}

I, significant increase; D, significant decrease; 0, lack of change; LP, lamina propria of the small intestine; AT, adipose tissue; DE, values in the diabetic mice treated with exercise; DH, values in diabetic mice treated with hyperbaric oxygenation; DEH diabetic mice treated with both exercise and hyperbaric oxygenation.

Adipose tissue. The initial levels of NO were similar in the adipose tissues of both the control and diabetic mice. The final levels were decreased in the control group $(\mathrm{P}<0.01)$, but were not significantly different in the diabetic mice, and none of the treatments altered NO levels significantly. The initial levels of 3NT were low in the control mice and were significantly higher in the diabetic mice $(\mathrm{P}<0.01)$. The final levels showed a moderate increase in the control group $(\mathrm{P}<0.05)$ and a decrease in the diabetic mice compared with the respective initial values $(\mathrm{P}<0.01)$, and none of the treatments significantly altered the $3 \mathrm{NT}$ levels. Compared with the adipose tissue of the control group, GSH levels were significantly lower in the diabetic mice $(\mathrm{P}<0.01)$, and the final levels were similar in both groups compared with their respective initial values, and only the combined treatment resulted in a significant decrease in the levels of GSH $(\mathrm{P}<0.01)$.

Spleen. The initials level of NO in the spleen tissue did not differ significantly between the two groups. The final NO levels were significantly lower in both groups compared with the respective initial values (both $\mathrm{P}<0.01$ ), and the final levels were higher in the diabetic group compared with the control group $(\mathrm{P}<0.01)$. All treatments reduced the concentration of NO (all $\mathrm{P}<0.05)$. The initial levels of $3 \mathrm{NT}$ were lower in the spleen of the control group compared with the diabetic group $(\mathrm{P}<0.01)$, and were significantly higher in the final measurement in both groups (both $\mathrm{P}<0.01$ ). The elevated initial $3 \mathrm{NT}$ levels in the diabetes group moderately decreased during phase $2(\mathrm{P}<0.01)$, 
leading to comparable final levels between the two groups. Treatment with exercise or HBO had no significant effect on $3 \mathrm{NT}$, but the combined treatment resulted in a significant increase $(\mathrm{P}<0.01)$. The initial levels of GSH were higher in the control group compared with diabetic group $(\mathrm{P}<0.01)$. The final levels decreased considerably in both groups compared with the respective initial values (both $\mathrm{P}<0.01$ ), reaching similar levels. The levels of GSH decreased with $\mathrm{HBO}$ alone $(\mathrm{P}<0.01)$, but increased with exercise and the combined treatment $(\mathrm{P}<0.01)$, suggesting that exercise predominated in the latter.

Brain. The initial levels of NO in brain tissue did not differ significantly between the control and diabetic mice, and there was a moderate significant decrease in the final values of NO only in the diabetic group $(\mathrm{P}<0.05)$. None of the treatments significantly altered the NO levels. The relatively low levels of $3 \mathrm{NT}$ in the control mice remained constant throughout the study. Conversely, the initial values of $3 \mathrm{NT}$ in the diabetic mice were significantly higher compared with the control group $(\mathrm{P}<0.01)$, and the final values in the diabetic group were significantly lower compared with the initial values in the diabetic mice $(\mathrm{P}<0.01)$. The final value of $3 \mathrm{NT}$ was higher in the diabetic mice compared with the control group and the treatments did not significantly affect the 3NT levels. The initial levels of GSH in the brain were not significantly affected by diabetes. The final levels were decreased in both groups (both $\mathrm{P}<0.01$ ), and were higher in the diabetic mice compared with the control mice $(\mathrm{P}<0.05)$. HBO increased the levels of GSH $(\mathrm{P}<0.01)$, and the combined treatment resulted in a decrease $(\mathrm{P}<0.01)$.

Pearson correlation. Regarding the initial levels of GSH and $3 \mathrm{NT}$ in all the evaluated tissues of the control mice, a significant negative correlation was observed when averaged across all tissues $(r=-0.734, \mathrm{P}=0.024)$ except liver (due to the high levels of GSH in this tissue), and this was not observed in the diabetic group. Under conditions of relative stability of NO levels in the control group, this stability indicates a direct antioxidant affect against peroxynitrite: A greater level of GSH corresponded to a lower level of $3 \mathrm{NT}$. There was a positive correlation observed between the initial levels of NO and the final levels of 3NT in the control group $(\mathrm{r}=0.641, \mathrm{P}=0.044)$ suggesting an increase in nitrosative stress: Higher initial levels of NO resulted in higher final levels of 3NT (data not shown).

A positive correlation was observed between the final levels of GSH and NO in the diabetic mice $(r=0.788, \mathrm{P}=0.007)$, suggesting a close association between these parameters in the pathological state as a correlation was not observed in the final values of the control tissues. The statistical analysis of the bivariate correlation of parameters in the tissues evaluated showed a positive correlation between NO and GSH in all tissues from the diabetic mice subjected to exercise (exercise alone: $\mathrm{r}=0.755, \mathrm{P}=0.012$; combination treatment, $\mathrm{r}=0.878$, $\mathrm{P}=0.01$ ). Table III shows which parameters were significantly altered in Table II.

The development of diabetes did not affect the level of NO in the tissues assessed except the aorta (Table IIIA). Conversely, induction of diabetes significantly increased the levels of $3 \mathrm{NT}$ in the majority of tissues (with a reduction in the muscle and lamina propria), while decreasing the levels of
GSH in adipose tissue and spleen, and increasing its levels in the aorta and lamina propria. The control and diabetes groups showed a drastic and significant decrease in the initial levels of NO and GSH in all tissues between the two phases of the study.

The positive effects of treatments against oxidative stress are reflected by a decrease in 3NT levels or an increase in GSH levels (4). Some treatments resulted in a protective effect against nitrosative stress in the diabetic mice in certain tissues (Table IIIB): Aorta, all treatments; and muscle, HBO. However, a negative effect (an increase in 3NT levels) was observed more frequently: Liver, all treatments; kidney, all treatments; intestinal lamina propria, all treatments; muscle, exercise and combined treatment; lung, exercise; and spleen, combined treatment. An increase in GSH levels were observed following all treatments in the muscle, heart and kidney, after exercise in the mucosa and spleen; after HBO only in the brain; and after combined treatment in the spleen.

\section{Discussion}

The results of the present study confirmed the successful establishment of a diet-induced diabetes mellitus type 2 model in mice. The age of mice is associated with the level of basal metabolism (lower in adults), and increases the likelihood of developing obesity based on diet (46-48). The possible effect of a change of basal metabolic levels in adult diabetic animals during the present study was eliminated by comparison of post-treatment levels of measured parameters to final levels of the diabetic group.

Oxidative stress served an important role in the pathogenesis of diabetes and complications linked to dysfunction of the endothelium. A possible initial mechanism of dysfunction of the endothelium may be associated with a reduction in the bioavailability of NO $(6,49,50)$. In the present study, the development of diabetes did not affect the levels of NO in the majority of tissues. Indirectly, the stability of NO during diabetes emphasizes the importance of its homeostasis in tissues of mice in the experimental model used. The development of diabetes drastically increased the levels of 3NT in the majority of tissues, which is indicative of nitrosative stress $(4,51)$, as it is a product of a reaction of peroxynitrite (derived from the reaction of NO with superoxide radicals) with the tyrosine residues of proteins. Conversely, the role of S-nitrosation in various signaling pathways associated with NO has also been discussed (25) and may be relevant for interpretation of the results of the present study considering the importance of S-nitrosation in the pathogenesis of diabetes. Principally, the effect of NO in the short-term is the vasodilatation of large conduit vessels that can modulate the redox state in tissues.

An increased production of superoxide in tissues is well-documented during the pathogenesis of diabetes $(7,9,16)$, suggesting increased elimination of NO by these superoxide radicals in the tissues of the diabetic mice. Therefore, the lack of difference in the levels of NO and elevated levels of nitrosative stress observed in the majority of tissues in the diabetic mice suggests that any increased elimination of NO by superoxides was compensated for by an increase in its production in this animal model. Excessive NO and superoxide production 
may disrupt the physiological balance between generation of peroxynitrite, antioxidant defense mechanisms and signaling pathways involving NO in the tissues of diabetic mice (4). GSH is a low-weight multifunctional molecule that also serves as the principal endogenous non-enzymatic antioxidant (15). In the present study, no changes in the levels of GSH were observed in the majority of tissues in the diabetic mice. In vivo studies have shown that the concentration of NO is closely associated with the synthesis of GSH in tissues. Increased production of NO inhibits the synthesis of GSH in rodent tissues, and a decreased production results in increased synthesis of GSH $(24,52)$. The stability observed in the levels of NO in the diabetic mice in the present study may therefore be associated with the stability in GSH levels in the majority of tissues in the present study.

In the initial values of the control group only, there was a significant negative correlation between the levels of GSH and $3 \mathrm{NT}$, and a positive correlation between the initial levels of NO and final levels of 3NT in all tissues. In the tissues of the control mice, the lower initial levels of nitrosative stress corresponded with an increase in antioxidant defense, which is manifested as higher levels of GSH. A high level of nitrosative stress resulted in a low level of GSH as the compensatory mechanisms were overwhelmed by excessive production of peroxynitrite and consequently 3NT. Compensatory mechanisms included increased production of GSH and/or its mobilization from the liver, where its concentration was in the $\mathrm{mM}$ range, compared with the $\mu \mathrm{M}$ concentrations observed in other tissues.

During phase 2 of the study, both the control and untreated diabetes groups displayed a similar decrease in the levels of $\mathrm{NO}$ and GSH in the majority of tissues, suggesting a possible age-related effect and thus may reflect a decrease in the basal levels of metabolism in adult mice. The lower initial levels of 3NT were not significantly altered or significantly increased in the majority of the tissues in the control group between the two phases, whereas the elevated initial levels of 3NT in the diabetic mice were unchanged or decreased between the two phases. The final levels of nitrosative stress (as determined based on 3 NT levels) for the control group indicated either stability or an increase in levels, and in the diabetic mice, the levels were either stable or decreased. In the majority of these tissues the final 3NT levels were higher in the diabetic mice compared with the control group. This difference coincided with a positive correlation between the final levels of GSH and NO in all tissues in the diabetic mice only, contradicting the known effect of $\mathrm{NO}$ on the synthesis of GSH in rodent tissues without diabetes $(24,53)$ which was also observed in the control group in the present study.

The role of NO-related mechanisms were examined in processes of adaptation to exercise in tissues (25). During a range of pathological processes and in all tissues and organs, NO was determined to be the primary molecule linked to the regulation of vascular endothelium tissue, and exercise may correct alterations in the bioavailability of this radical. Enzymatic production of NO by endothelial nitric oxide synthase has a regulatory role in endothelial dysfunction (54) and NO participates in vascular adaptation to exercise $(55,56)$. Therefore, the association between NO and different nitric oxide synthases in diabetic tissues requires examination.

Studies have described the protective effect of $\mathrm{HBO}$ pre-treatment against oxidative stress produced by distinct factors in different animal models, including experimental damage to the rat spinal cord (27), cerebral ischemia-reperfusion (28), experimental myocardial infarct (53), and the effect of ultraviolet light on mouse skin and liver (29). HBO pretreatment is also reported to reduce the levels of indicators of oxidative stress in rat thoracic aorta, including NO (31) and to decrease oxidative stress in rat lungs (30). In the present study, exercise and HBO did not affect the levels of NO in the majority of diabetic tissues, but the levels of nitrosative stress (3NT) and GSH were increased in the majority of tissues, and contrary to the control group, where a negative correlation was observed between these parameters. An increase in the levels of GSH was interpreted as a positive effect in relation to the redox equilibrium in tissues.

All treatments did not alter or increase the levels of GSH in muscle, liver heart, kidney, lung and brain tissues (except after HBO alone in the lung, or after combined treatment in the brain) suggesting a positive effect on the redox equilibrium of tissues. HBO alone increased the levels of GSH in the brain and decreased the levels in the lung. Conversely, exercise alone resulted in changes in GSH levels in the small intestine, where an increase was observed in the mucosa and a decrease was observed in the lamina propria, and this may be reflective of redistribution of GSH between different intestinal regions. The combination treatment showed a similar effect on GSH levels as exercise in the majority of tissues.

Increased or unchanged levels of GSH by all treatments corresponded to an increase or unchanged levels of nitrosative stress. It is possible that the elevated levels of nitrosative stress facilitated the redistribution of GSH from liver to other tissues. As the final level of GSH in liver of diabetic mice was $\geq 80$-fold higher compared with other tissues, alterations in the level of GSH in the liver may have gone unnoticed. In our previous study $\mathrm{HBO}$-induced a notable decrease in the basal levels of GSH in the liver which coincided with an increase in the muscle, brain, small intestine and adipose tissue, without a significant change in the grade of GSH oxidation in the exercised mice (57). That is, the change in GSH without a change in the oxidation of GSH following $\mathrm{HBO}$, suggested that GSH was redistributed from the liver to the other, reflecting an improvement in the antioxidant capability of these tissues.

In conclusion, the development of diabetes in this animal model caused nitrosative stress in the majority of tissues without affecting GSH levels in the majority tissues, and all treatments resulted in an increase or stabilization of GSH levels in the muscle, liver, heart, kidney, lung and brain which coincided with an increase or stabilization in nitrosative stress in the same tissues.

\section{Acknowledgements}

We would like to thank our students Dr Pérez Castro CC, Franco-Valdillo A and Dr Toledo-Blas Mirelle for their assistance during the study.

\section{Funding}

The present study was funded by SIP and COFAA of National Polytechnic Institute. 


\section{Availability of data and materials}

The datasets used and/or analyzed during the present study are available from the corresponding author on reasonable request.

\section{Authors' contributions}

All authors performed the experiments. AK, GGB and $\mathrm{MDCCH}$ performed the analysis and wrote the manuscript. LRGC and ELP performed the analysis and interpreted the data. All authors read and approved the final the manuscript.

\section{Ethics approval and consent to participate}

The study protocol was reviewed and approved by the Institutional Laboratory Use and Care Committee of the High Medical School, National Polytechnic Institute (Mexico City, Mexico; approval no. 02/28-08-2015).

\section{Patient consent for publication}

Not applicable.

\section{Competing interests}

The authors declare that they have no competing interests.

\section{References}

1. Lo Faro ML,Fox B, Whatmore JL, Winyard PG and Whiteman M Hydrogen sulfide and nitric oxide interactions in inflammation. Nitric Oxide 41: 38-47, 2014.

2. Alderton WK, Cooper CE and Knowles RG: Nitric oxide synthases: Structure, function and inhibition. Bichem J 357: 593-615, 2001.

3. Zhang Z, Naughton D, Winyard PG, Benjamin N, Blake DR and Symons MC: Generation of nitric oxide by a nitrite reductase activity of xanthine oxidase: A potential pathway for nitric oxide formation in the absence of nitric oxide synthase activity. Biochem Biophys Res Commun 249: 767-772, 1998.

4. Beckman JS and Koppenol WH: Nitric oxide, superoxide, and peroxynitrite: The good, the bad, and ugly. Am J Physiol 271: 1424-1437, 1996

5. Chen JY, Ye ZX, Wang XF, Chang J, Yang MW, Zhong HH, Hong FF and Yang SI: Nitric oxide bioavailability dysfunction involves in atherosclerosis. Biomed Pharmacother 97: 423-428, 2018.

6. Delamea BS, Leitão CB, Freadman R and Canani LH: Nitric oxide system and diabetic nephropatju. Diabetol Metab Syndr 6 : $17-21,2014$

7. Yuan T, Yang T, Chen H, Fu D, Hu Y, Wang J, Yuan Q, Yu H, $\mathrm{Xu} \mathrm{W}$ and Xie $\mathrm{X}$ : New insights into oxidative stress and inflammation during diabetes mellitus-accelerated atherosclerosis Redox Biol 20: 247-260, 2019.

8. Low Wang CC, Hess CN, Hiatt WR and Goldfin AB: Clinical update: Cardiovascular disease in diabetes mellitus: Atherosclerotic cardiovascular disease and heart failure in type 2 diabetes mellitus mechanisms, management, and clinical considerations. Circ 133: 2459-2502, 2016

9. Wang W, Shang C, Zhang W, Jin Z, Yao F, He Y, Wang B, Li Y, Zhang $\mathrm{J}$ and Lin R: Hydroxityrosol regulates oxidative stress and NO production through SIRT1 in diabetic mice and vascular endothelial cells. Phytomed 52: 206-215, 2019.

10. Assman TS, Brondani LA, Bouças AP, Rheinheimer J, de Souzza BM, Canani LH, Bauer AC and Crispim D: Nitric oxide levels in patients with diabetes mellitus: A systematic review and meta-analysis. Nitric Oxide 61: 1-9, 2016.

11. Millerbon FJ, Gutterman DD, Rios CD, Heistad DD and Davidson BI: Superoxide production in vascular smooth muscle contributes to oxidative stress and impaired relaxation in atherosclerosis. Circ Res 82: 1298-1303, 1998.
12. Tabit CE, Chung WB, Hamburg NM and Vita JA: Endothelial dysfunction in diabetes mellitus: Molecular mechanisms and clinical implications. Rev Endocr Metab Disord 11: 61-74, 2010.

13. Cosby K, Partovi KS, Crawford JH. Patel RP, Reiter CD, Martyr S, Yang BK, Waclawiw MA, Zalos G, Xu X, et al: Nitrite reduction to nitric oxide by deoxyhemoglobin vasodilates the human circulation. Nat Med 9: 1498-1505, 2003.

14. Totzeck M, Hengen-Cotta UB, Luedike P, Berenbrink M, Klare JP, Steinhoff HJ, Semmler D, Shiva S, Williams D, Kipar A, et al: Nitrite regulates hypoxic vasodilatation via myoglobin-dependent nitric oxide generation. Circ 126: 325-334, 2012 .

15. Wu G, Fang YZ, Yang S, Lupton JR and Turner ND: Glutathione metabolism and its implications for health. J Nutr 134:489-492, 2004.

16. Sundaram RK, Bhaskar A, Vijayalingam S, Viswanathan M, Mohan R and Shanmugasundaram KR: Antioxidant status and lipid peroxidation in type II diabetes mellitus with and without complications. Clin Sci (Lond) 90: 255-260, 1996.

17. Nguyen D, Hsu JW, Jahoor F and Sekhar RV: Effect of increasing glutathione with cysteine and glycine supplementation on mitochondrial fuel oxidation, insulin sensitivity, and body composition in older HIVinfected patients. J Clin Endocrinol Metab 99: 169-177, 2014.

18. Sekhar RV, McKay SV, Patel SG, Guthikonda AP, Reddy VT, Balasubramanyam A and Jahoor F: Glutathione synthesis is diminished in patients with uncontrolled diabetes and restored by dietary supplementation with cysteine and glycine. Diabetes Care 34: 162-167, 2011.

19. Lagman M, Ly J, Saing T, Kaur Singh M, Vera Tudela E, Morris D, Chi P-T, Ochoa C, Sathananthan A and Venketaran V: Investigating the causes for decreased levels of glutathione in individuals with type II diabetes. PLoS One 10: e0118436, 2015.

20. Lutchmansingh FK, Hsu JW, Bennett FI, Badaloo AV, McFarlaneAnderson N, Gordon-Strachan GM, Wright-Pascoe RA, Jahoor F, Michael S and Boyne MS: Glutathione metabolism in type 2 diabetes and its relationship with microvascular complications and glycaemia. PLoS One 13: e0198626, 2018.

21. Parsanathan R and Jain SK: Glutathione deficiency alters the vitamin D-metabolizing enzymes CYP27B1 and CYP24A1 in human renal proximal tubule epithelial cells and kidney of HFD-fed mic. Free Radic Biol Med 131: 376-381, 2019.

22. Ilyer AKV, Rojanasakul Y and Azad N: Nitrosothiols signaling and protein nitrosation in cell death. Nitric Oxide 42: 9-18, 2014.

23. Ganzarolli de Oliveira M: S-nitrosothiols as platforms for topical nitric oxide delivery. Basic Clin Pharmacol Toxicol 119: 49-56, 2016.

24. Payabvash S, Ghahremani MH, Goliaei A, Mandegary A, Shafaroodi H, Amanlou M and Dehpour AR: Nitric oxide modulates glutathione synthesis during endotoxemia. Free Rad Biol Med 41: 1817-1828, 2006.

25. Surh F, Gehlert S, Grau M and Bloch W: Skeletal muscle function during exercisefine-tuning of diverse subsystems by nitric oxide. Int J Mol Sci 14: 7109-7139, 2013.

26. Yaribeygi HY, Butler AE and Sahebkar A: Aerobic exercise can modulate the underlying mechanisms involved in the development of diabetic complications. J Cell Physiol 234: 12508-12515, 2019.

27. Kahraman S, Düz B, Kayali H, Korkmaz A, Öter S, Aydin A and Sayal A: Effects of methylprednisolone and hyperbaric oxygen on oxidative status after experimental spinal cord injury: Comparative study in rats. Neurochem Res 32: 1547-1551, 2007.

28. Cheng O, Ostrowski RP, Wu B, Liu W, Chen C and Zhang JH: Cyclooxygenase 2 mediated hyperbaric oxygen preconditioning in the rat model of transient global cerebral ischemia. Stroke 422: 484-490, 2011.

29. Fuller AM, Giardina C, Hightower LE, Perdrizet GA and Tierney CA: Hyperbaric oxygen preconditioning protects skin from UV-A damage. Cell Stress Chaperones 8: 97-107, 2013.

30. Feng Y, Zhang Z, Li Q, Li W, Xu J and Cao H: Hyperbaric oxygen preconditioning protects lung against hyperoxic acute lung injury via heme oxygenase-1 induction. Biochem Biophys Res Comm 459: 549-554, 2015.

31. Guevara-Balcazar G, Lara-Padilla E, Kormanovski A, RamirezSanchez I, Castillo-Henkel EF and Castillo-Hernandez MC: Changes in oxidative stress and vascular reactivity of thoracic and abdominal rat aorta with different periods of exposure to hyperbaric oxygenation. Int J Pharmac 11: 611-617, 2015.

32. Thom SR: Oxidative stress is fundamental to hyperbaric oxygen therapy. J Appl Physiol (1985) 106: 988-995, 2009. 
33. Castillo-Hernandez MC, Lara-Padilla E, Kormanovski A, Perez-Tuñon JG, Lopez-Calderon EM and Guevara-Balcazar G: Normalization of QRS segment, blood pressure and heartbeat in an experimental model of amintriptyline intoxication in rats following hyperbaric oxygenation therapy. Int J Pharmac 11: 508-512, 2015.

34. Buras JA, Sthal GL, Svoboda KK and Reenstra WR: Hyperbaric oxygen down-regulates ICAM-1 expression induced by hypoxia and hypoglycemia: The role of NOS. Am J Physiol Cell Physiol 278: 292-302, 2000.

35. Cabigas BP, Su J, Hutchins W, Shi Y, Schaefer RB, Recinos RF, Nilakantan V, Kindwall E, Niezgoda JA and Baker JE: Hyperoxic and hyperbaric-induced cardioprotection: Role of nitric oxide synthase 3. Cardiovasc Res 72: 143-151, 2006.

36. Buerk DG: Nitric oxide regulation of microvascular oxygen. Antiox Red Signal 9: 829-843, 2007.

37. Thibault L: Animal models of dietary-induced obesity. In animal models for the study of human disease. pp. 277-303, 2013.

38. Sah SP, Singh B, Choudhary S and Kumar A: Animal models of insulin resistance: A review. Pharmacol Rep 68: 1165-1177, 2016

39. Ravi Kiran T, Subramanyam MV and Asha Devi S: Swim exercise training and adaptations in the antioxidant defense system of myocardium of old rats: Relation to swim intensity and duration. Comp Biochem Physiol B Biochem Mol Biol 137: 187-196, 2004

40. Nacao C, Ookawara S, Kizaki T, Oh-Ishi S, Miyazaki H, Haga S, Sato Y, Ji LL and Ohno H: Effects of swimming training on three superoxide dismutase isoenzymes in mouse tissue. J Appl Physiol (1985) 88: 649-654, 2000.

41. Jung UJ and Choi MS: Obesity and its metabolic complications: The role of adipokines and the relationship between obesity, inflammation, insulin resistance, dyslipidemia and nonalcoholic fatty liver disease. Int J Mol Sci 15: 6184-6223, 2014.

42. Rogers P and Webb GP: Estimation of body fat in normal and obese mice. Brit J Nutr 43: 83-87, 1980.

43. Weyer C, Bogardus C, Mott DM and Pratley RE: The natural history of insulin secretory dysfunction and insulin resistance in the pathogenesis of type 2 diabetes mellitus. J Clin Invest 104 787-794, 1999

44. Defronzo RA, Eldor R and Abdul-Ghani M: Pathophysiologic approach to therapy in patients with newly diagnosed type 2 diabetes. Diab Care 36 (Suppl 2): S127-S138, 2013.

45. Muthulakshmi S and Saravanan R: Protective effects of azelaic acid against high-fat diet-induced oxidative stress in liver, kidney and heart of C57BL/6J mice. Mol Cell Biochem 377: 23-33, 2013.
46. Vickers SP, Jackson HC and Cheetham SC: The utility of animal models to evaluate novel anti-obesity agents. Br J Pharmacol 164: 1248-1262, 2011.

47. Makarov M and Makarov V: Diet-induced models of metabolic disturbances. Report 2: Experimental obesity. Lab Animals Sci 2: 12-17, 2018

48. Rosini TC, da Silva ASR and de Moraes C: Diet-induced obesity: Rodent model for the study of obesity related disorders. Rev Assoc Med Bras (1992) 58: 383-387, 2012 (In English, Portuguese).

49. Schmidt HH and Walter U: NO at work. Cell 78: 919-925, 2004.

50. Napoli $\mathrm{C}$ and Ignarro LJ: Nitric oxide and pathogenic mechanisms involved in the development of vascular diseases. Arch Pharmacol Res 32: 1103-1108,2009.

51. Davies MJ, Fu S, Wang H and Dean RT: Stable markers of oxidant damage to proteins and their application in the study of human disease. Free Radic Biol Med 27: 1151-1163, 1999.

52. Minamiyama Y, Takemura S, Koyama K, Yu H, Miyamoto M and Inone M: Dynamic aspects of glutathione and nitric oxide metabolism in endotoxemic rats. Am J Physiol 271: G575-G581, 1996.

53. Sun Q, Liu Y, Sun X and Tao H: Anti-apoptotic effect of hyperbaric oxygen preconditioning on a rat model of myocardial infarction. J Surg Res 171: 41-46, 2011.

54. Nisoli E, Clementi E, Paolucci C, Cozzi V, Tonello C, Sciorati C, Bracale R, Valerio A, Francolini M, Moncada S and Carruba MO: Mitochondrial biogenesis in mammal: The role of endogenous nitric oxide. Science 299: 896-899, 2003.

55. Miller MW, Knaub LA, Olivera-Fragoso LF, Keller AC. Balasubramaniam V, Watson PA and Reusch JEB: Nitric oxide regulates vascular adaptive mitochondrial dynamics. Am J Physiol Heart Circ Physiol 304: H1624-H1633, 2013.

56. Whal MP, Scalzo RL, Regensteiner JG and Reusch JEB: Mechanisms of aerobic exercise impairment in diabetes: A narrative review. Front Endocrinol (Lausanne) 9: 181, 2018.

57. Kormanovski A, Castillo-Hernández MC, Guevara-Balcazar G, Pérez T and Lara-Padilla E: Gender difference in nitric oxide and antioxidant response to physical stress in tissues of trained mice after hyperbaric oxygen preconditioning: Part 2. Physiol Pharmac 23: 309-321, 2019.

This work is licensed under a Creative Commons Attribution-NonCommercial-NoDerivatives 4.0 International (CC BY-NC-ND 4.0) License. 